\title{
Static analysis of a reinforced bimodulus beam on elastic foundation
}

\author{
Alexey Beskopylny ${ }^{1, *}$, Elena Kadomtseva ${ }^{1}$, and Grigory Strelnikov ${ }^{1}$ \\ ${ }^{1}$ Don State Technical University, pl. Gagarin, 1, Rostov-on-Don, 344010, Russia
}

\begin{abstract}
The work considers a reinforced concrete beam made of bimodule material on an elastic Winkler base. The influence of the number of reinforcing bars located in the stretched zone on the maximum normal stress and the maximum deflection is numerically investigated. The choice of the cross-section dimensions, such as the width and thickness of the flanges, the height of the beam, causes particular difficulties and is limited by the need to determine specific ratios of I-beam sizes. Numerical investigation allows analyzing the influence of the width of the I-beam flange that is in contact with the elastic base on the maximum tensile stresses and maximum deflection. The effect on the stressed-deformed state of the various cross-section forms is carried out in work for an Ibeam, a rectangle, and a T-beam. Related to the fact that for many building materials the elastic moduli for tension and compression differ, it became necessary to determine the influence of material heterogeneity on the stress-strain state of the elements of building structures. In particular, the widespread use of reinforced concrete beams as elements of band foundations requires a refinement of the stress-strain state study taking into account the heterogeneity of concrete.
\end{abstract}

\section{Introduction}

In the construction of widely used structural elements based on an elastic base. Such an example is the closely spaced columns, beams or crossbars of frames, which are based on the ground or piles. In this paper we consider an elastic foundation means mostly the natural soil, or pile foundation.

At present, three methods are mainly used to calculate structures on an elastic basis: 1) methods based on the Winkler base model; 2) methods based on the theory of elastic halfspace; 3) methods based on the combined models of the elastic foundation.

The model of the Winkler base gives the results closest to the experimental ones, which is proved by the experiments carried out with rigid stamps [1,2], dynamic indentation [3], experiments with finite stiffness beams [4,5]. In this paper, we consider an arbitrarily loaded, arbitrarily supported reinforced concrete beam made of bimodule material on an elastic Winkler base. The influence of the number of reinforcement bars located in the stretched zone on the maximum normal stress and maximum deflection is numerically investigated.

\footnotetext{
${ }^{*}$ Corresponding author: besk-an@ya.ru
} 
Selection of the analytical cross-sectional dimensions of width and thickness of the shelves, the height of the wall causes some difficulties and is limited by the need of setting a specific ratio of the size of the I-beam. The analytical statement and numerical study [6,7] allows us to analyze the effects of the shelf width of the I-beam in contact with the elastic base on the maximum normal stresses and maximum deflection.

Because for many building materials elastic modulus of tension and compression are different, it became necessary to determine the effect of heterogeneity of the material on the stress - strain state of the elements of building structures. In particular, the widespread use of reinforced concrete beams as elements of strip foundations requires clarification of the study of stress-strain state, taking into account the properties of heterogeneity of concrete.

In construction area, these methods are widely used for design projects, diagnostics and monitoring of structures. These are non-destructive tests based on indentation methods that used for metal structures $[8,9]$, determination of the rheological parameters for the creep models [10].

\section{Problem statement and equipment}

A heterogeneous model of a reinforced beam is represented as a beam consisting of two layers: stretched, compressed and reinforcing bars. Considering the beam as statically indeterminate, we obtain

$$
\begin{gathered}
M_{y}=\int_{A} \sigma z d A=\int_{A_{p}} \sigma_{p} z d A+\int_{A_{c}} \sigma_{c} z d A+\int_{A_{a}} \sigma_{a} z d A=M_{y_{p}}+M_{y_{c}}+M_{y_{a}} \\
\frac{1}{\rho}=\frac{1}{\rho_{p}}=\frac{1}{\rho_{c}}=\frac{1}{\rho_{a}}
\end{gathered}
$$

Where $M_{y}, \frac{1}{\rho}$ - bending moment and beam curvature; $M_{y_{p}}, \frac{1}{\rho_{p}}$ - bending moment and curvature of the beam of the stretched zone; $M_{y_{c}}, \frac{1}{\rho_{c}}$ - bending moment and curvature of the beam compression area; $M_{y_{a}}, \frac{1}{\rho_{a}}$ - the bending moment and the curvature of the rods.

Equation (2) expresses the condition of compatibility of heterogeneous beam deformations. The normal stresses given (2) have the form

$$
\sigma_{p}=\frac{E_{p} z}{\rho}, \sigma_{c}=\frac{E_{c} z}{\rho}, \sigma_{a}=\frac{E_{a} z}{\rho}
$$

Here $\sigma_{p}, A_{p}$ - normal stress and the cross-sectional area of the beam stretched zone, $\sigma_{c}$, $A_{c}$ - the normal stress and the cross-sectional area of the beam in the compression zone, the compression $\sigma_{a}, A_{A}$ - normal stress and the cross-sectional area of rebar. form

Using the equilibrium condition (1) $\Sigma \boldsymbol{M}_{\boldsymbol{y}}=0$ expressed regarding normal stresses has the 


$$
M_{y}=\int_{A} \sigma z d A=\int_{A_{p}} \sigma_{p} z d A+\int_{A_{c}} \sigma_{c} z d A+\int_{A_{a}} \sigma_{a} z d A=M_{y_{p}}+M_{y_{c}}+M_{y_{a}}
$$

Substituting the normal stress (3) into (4) we obtain the formula of curvature to the neutral axis for a beam from a bimodal material

$$
M_{y}=\frac{1}{\rho}\left(E_{p} J_{y}^{p}+E_{c} J_{y}^{c}+E_{a}\left(n_{p}\left(J_{y_{1}}^{p}+A_{a}^{p} c_{p}^{2}\right)+n_{c}\left(J_{y_{1}}^{c}+A_{a}^{c} c_{c}^{2}\right)\right)\right)
$$

From (5) we obtain a formula for determining the radius of curvature for a reinforced beam of heterogeneous material

$$
\frac{1}{\rho}=\frac{M_{y}}{D}=\frac{M_{y_{p}}+M_{y_{c}}+M_{y_{a}}}{D}
$$

Where $D=E_{p} J_{y}^{p}+E_{c} J_{y}^{c}+E_{a}\left(n_{p}\left(J_{y_{1}}^{p}+A_{a}^{p} c_{p}^{2}\right)+n_{c}\left(J_{y_{1}}^{c}+A_{a}^{c} c_{c}^{2}\right)\right), D$ - reduced stiffness of a beam made of heterogeneous material; $E_{p}, E_{c}$ elastic modules of the material in the areas of tension and compression; $E_{a}$ the modulus of elasticity of reinforcement; $J_{y}^{p}$ the moment of inertia of the part of the cross section that lies in the stretching area, relative to the neutral axis; $J_{y}^{c}$ the moment of inertia of the cross-section that lies in the compression zone, relative to the neutral axis; $J_{y_{1}}^{p}$ the moment of inertia of the cross-section of the valve, which lies in the stretching zone, relative to its own central axis; $J_{y_{1}}^{c}$ the moment of inertia of the cross-section of the valve, which lies in the compression zone, relative to its own central axis; $n_{p}, n_{c}$ number of reinforcement bars in tension and compression zones; $A_{a}^{p}, A_{a}^{c}$ the cross-sectional area of the valve in the areas of tension and compression; $c_{p}, c_{c}$ distance from reinforcement in the areas of tension and compression to the neutral axis.

Comparing formulas (4) and (5), we obtain the reduced stiffness expression for reinforced beams of heterogeneous material $D$ in form (6). The position of the neutral line is determined by the equation (7) obtained from the static equation-the projection of internal forces on the axis of the $\operatorname{rod} \Sigma F_{x}=0$

$$
E_{p} S_{y}^{p}+E_{c} S_{y}^{c}+E_{a}\left(n_{p} A_{a}^{p} c_{p}+n_{c} A_{a}^{c} c_{c}\right)=0
$$

Where $S_{y}^{p}, S_{y}^{c}$ static moments of the area of the stretched and compressed zone of the cross-section, relative to the neutral axis.

The formulas of normal stresses taking into account (3), (4) have the form

$$
\sigma_{p}=\frac{E_{p} M_{y}}{D} z, \sigma_{c}=\frac{E_{c} M_{y}}{D} z, \sigma_{a}=\frac{E_{a} M_{y}}{D} z .
$$

To determine the deflection $v(x)$, bending moment $M_{\mathrm{y}}$, the known formulas for forces and displacements for the beam on an elastic base, expressed in terms of the Krylov function, were used $Y_{1}(\xi), Y_{2}(\xi), Y_{3}(\xi), Y_{4}(\xi)$ 


$$
\begin{aligned}
D \mathrm{v}(x)= & D v_{0} \cdot Y_{1}\left(\frac{x}{L}\right)+D \varphi_{0} \cdot L Y_{2}\left(\frac{x}{L}\right)-\mathrm{M}_{0} L^{2} Y_{3}\left(\frac{x}{L}\right)-\mathrm{Q}_{0} L^{3} Y_{4}\left(\frac{x}{L}\right)-\sum m_{i} L^{2} Y_{3}\left(\frac{x-a_{i}}{L}\right)+ \\
& +\sum q_{i} \frac{L^{4}}{4}\left[1-Y_{1}\left(\frac{x-c_{i}}{L}\right)\right]-\sum q_{i} \frac{L^{4}}{4}\left[1-Y_{1}\left(\frac{x-d_{i}}{L}\right)\right]+\sum F_{i} L^{3} Y_{4}\left(\frac{x-b_{i}}{L}\right) \\
M(x)= & \alpha L^{2} \mathrm{v}_{0} \cdot Y_{3}\left(\frac{x}{L}\right)+\varphi_{0} \alpha L^{3} \cdot Y_{4}\left(\frac{x}{L}\right)+\mathrm{M}_{0} Y_{1}\left(\frac{x}{L}\right)+\mathrm{Q}_{0} L Y_{2}\left(\frac{x}{L}\right)+\sum m_{i} Y_{1}\left(\frac{x-a_{i}}{L}\right)- \\
& -\sum q_{i} L^{2} Y_{3}\left(\frac{x-c_{i}}{L}\right)+\sum q_{i} L^{2} Y_{3}\left(\frac{x-d_{i}}{L}\right)-\sum F_{i} L Y_{2}\left(\frac{x-b_{i}}{L}\right) ;
\end{aligned}
$$

Where $v_{0}$ the deflection at the origin; $\varphi_{0}$ the angle of rotation at the origin; $M_{0}$ the bending moment at the origin; $Q_{0}$ the shear force at the origin; $m_{i}$ concentrated moment applied at $a_{i}$ distance from the left end of the beam; $F_{i}$ the concentrated force applied at the distance $b_{i}$ from the left end of the beam; $q_{i}$ the intensity of the uniformly distributed load on the section that starts at the distance $c_{i}$ from the left end of the beam and ends at the distance $d_{i}$ from the left end of the beam; $L=\sqrt[4]{\frac{4 D}{\alpha}}, \xi=\frac{x}{L}, \alpha=k_{0} b, k_{0}$ coefficient of elasticity of the base, $b$ section width.

\section{Results and discussion}

A numerical study was conducted for a simply supported beam on elastic Winkler the basis of I-and t-cross section, made of reinforced foam concrete in the middle loaded with a concentrated force. The material of the beam has the property of modularity, i.e. modulus of elasticity in tension and compression are different, but the material is isotropic. It has proven $[4,5]$ that for such materials hypotheses and formulas of resistance of materials and the theory of elasticity are true. Characteristics of fiber foam concrete $E_{c}=2.25 \cdot 10^{3} \mathrm{MPa}$; $E_{p}=5.0 \cdot 10^{3} \mathrm{MPa}$ (Figure 1). Characteristics of reinforcement bars $E_{a}=2.06 \cdot 10^{5} \mathrm{MPa}, d_{p}=12$ $\mathrm{mm} ; d_{c}=8 \mathrm{~mm}$.
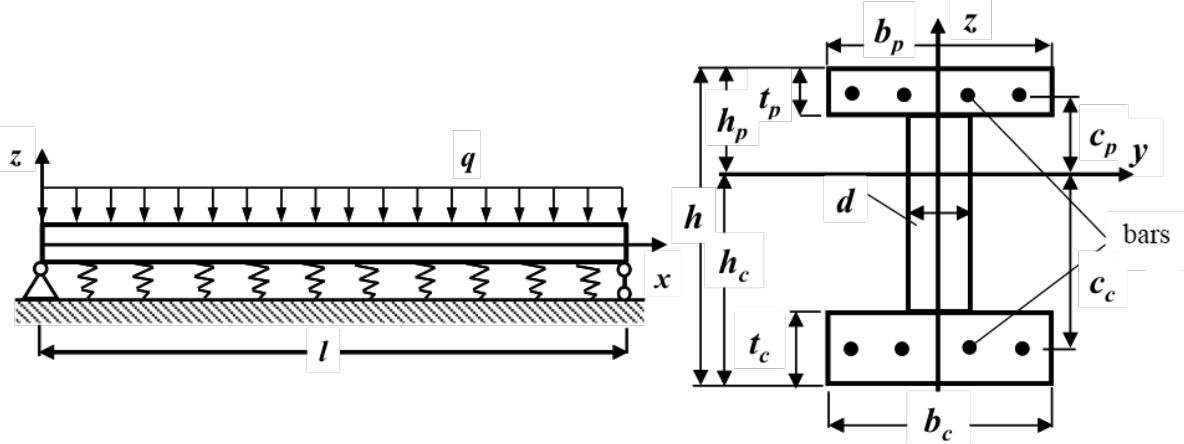

Fig. 1. Scheme of the beam on elastic Winkler base of I-beam and t-cross section

We consider I-beam with following characteristics: $l=4 \mathrm{~m}, h=89.0 \mathrm{~cm}, b_{\mathrm{p}}=28.0 \mathrm{~cm}$, $t_{\mathrm{p}}=15.0 \mathrm{~cm}, d_{\mathrm{c}}=12.0 \mathrm{~cm}, b_{\mathrm{c}}=28.0 \mathrm{~cm}, t_{\mathrm{c}}=8.0 \mathrm{~cm}$. The load is evenly distributed along the entire length of the beam $q=100 \mathrm{kN} / \mathrm{m}$, modulus of elasticity of foundation $k_{0}=100 \mathrm{MPa} / \mathrm{m}$ (Figure 2). 

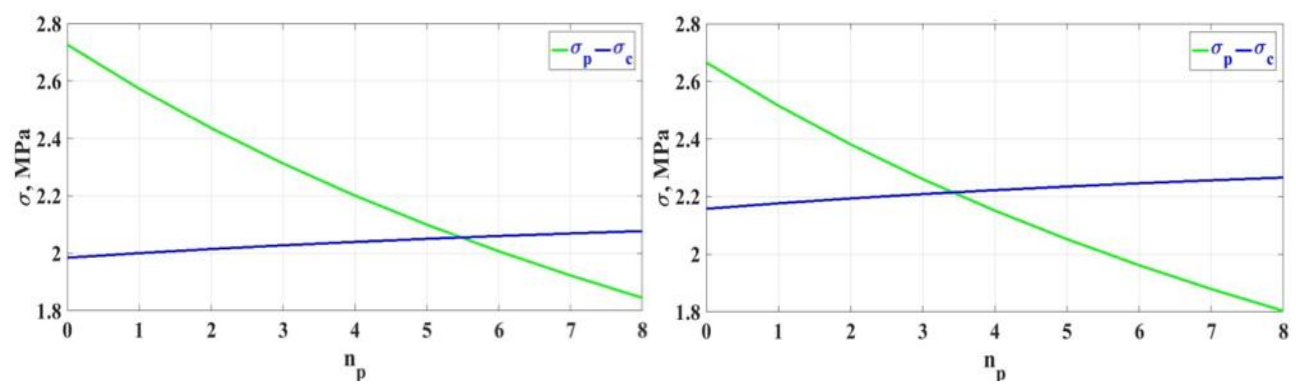

Fig. 2. Stresses of a bimodule I-beam on an elastic base depending on the number of rods $n_{\mathrm{c}}$ in the compression zone $E_{\mathrm{p}}=5000 \mathrm{MPa}, E_{\mathrm{c}}=2250 \mathrm{MPa}: n_{\mathrm{c}}=2$ (left), $n_{\mathrm{c}}=0$ (right)

Taking into account the bimodularity, the maximum tensile normal stresses arising in the crosssection of the I-beam decrease with the increase in the number of reinforcement bars in the stretched zone both in the presence of the reinforcement in the compressed zone and its absence, while the compressive stresses increase. When the rods are located in the compressed zone, the tensile normal stresses increase in absolute value, and the compressive normal stresses decrease in comparison with the corresponding stresses in the absence of reinforcement in the compressed zone.

The qualitative picture of the dependence of the maximum normal stresses on the number of reinforcement bars and their location without taking into account bimodularity does not change. However, without bimodality, the normal compressive stresses at any number of reinforcement bars are higher than the normal tensile stresses. The absolute value of the normal compressive stress, taking into account the bimodality of $38 \%$ more, and the normal tensile stress is more by $74 \%$ than the corresponding normal stress, excluding bimodularity (Figure 3).

Figure 4 shows a study of the dependence of the normal stresses arising in the beam of the tsection without taking into account the bimodularity of the material. The cross-sectional area of the tbeam is half the cross-sectional area of the I-beam. The normal tensile stresses in the t-beam and Ibeam beams are practically the same, and the compressive normal stresses in the t-beam are $29 \%$ higher than in the I-beam.
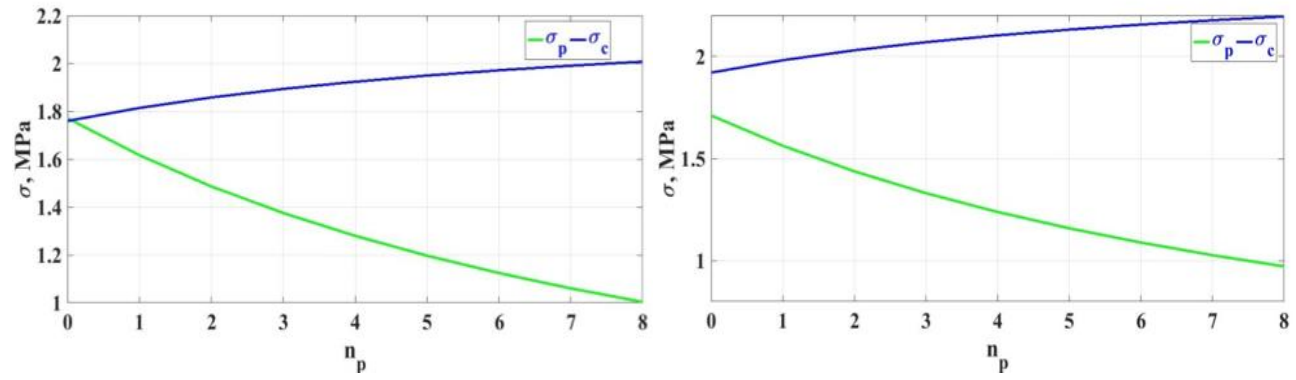

Fig. 3. Stresses of an I-beam (without bimodulus) on an elastic base depending on the number of rods $n_{\mathrm{c}}$ in the compression zone $E_{\mathrm{p}}=2250 \mathrm{MPa}, E_{\mathrm{c}}=2250 \mathrm{MPa}: n_{\mathrm{c}}=2$ (left), $n_{\mathrm{c}}=0$ (right) 

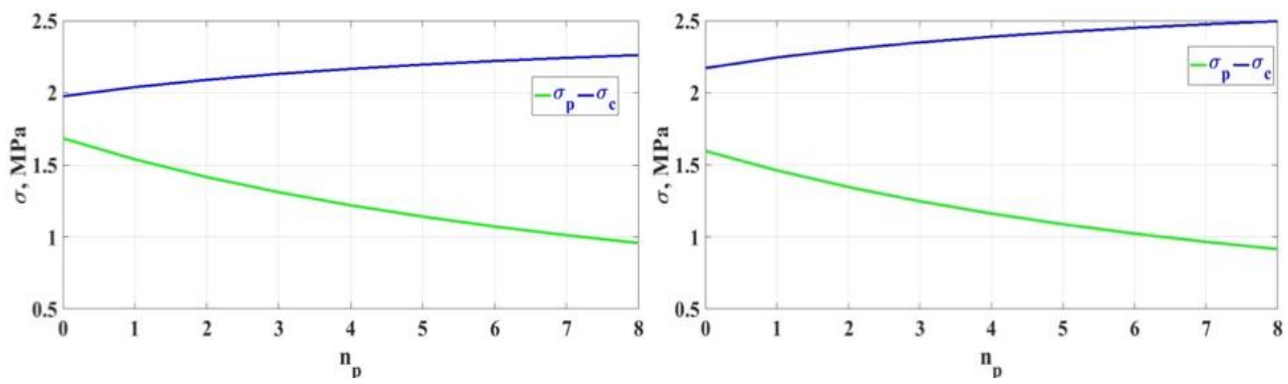

Fig. 4. Stresses of a t-beam (without bimodulus) on an elastic base depending on the number of rods $n_{\mathrm{c}}$ in the compression zone $E_{\mathrm{p}}=2250 \mathrm{MPa}, E_{\mathrm{c}}=2250 \mathrm{MPa}: n_{\mathrm{c}}=2$ (left), $n_{\mathrm{c}}=0$ (right)
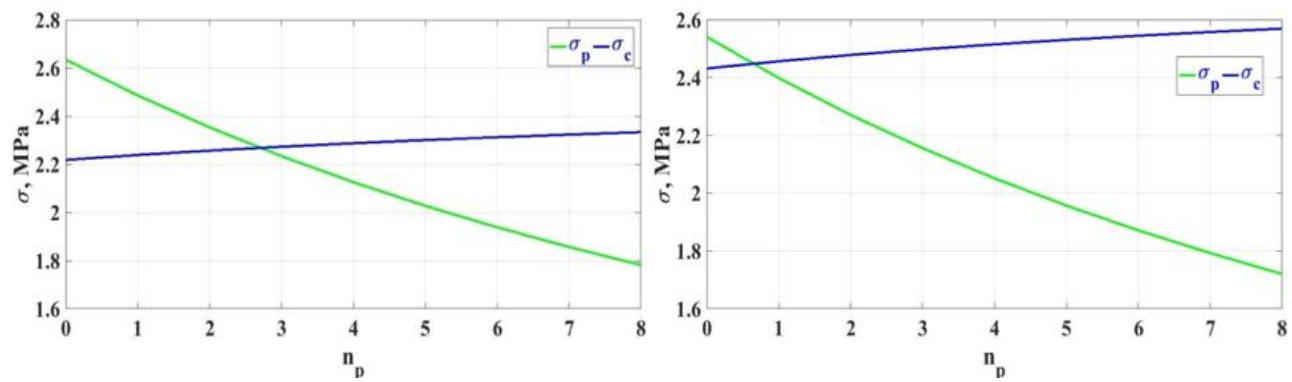

Fig. 5. Stresses of a bimodule t-beam on an elastic base depending on the number of rods $n_{\mathrm{c}}$ in the compression zone $E_{\mathrm{p}}=5000 \mathrm{MPa}, E_{\mathrm{c}}=2250 \mathrm{MPa}: n_{\mathrm{c}}=2$ (left), $n_{\mathrm{c}}=0$ (right)

As can be seen from figure 5 , the bimodality of the t-beam has almost no effect on the amount of compressive stresses, while the tensile stresses increase by $69 \%$.

When the width of the lower shelf of the brand is increased by $7 \%$ (the shelf in contact with the elastic base), the tensile normal voltage is reduced by $9 \%$, the compressive normal voltage is reduced by $7 \%$. Increasing the width of the bottom shelf by $20 \%$ reduces normal stress: compressive by $18 \%$, stretching by $28 \%$ (Figure 6 ).
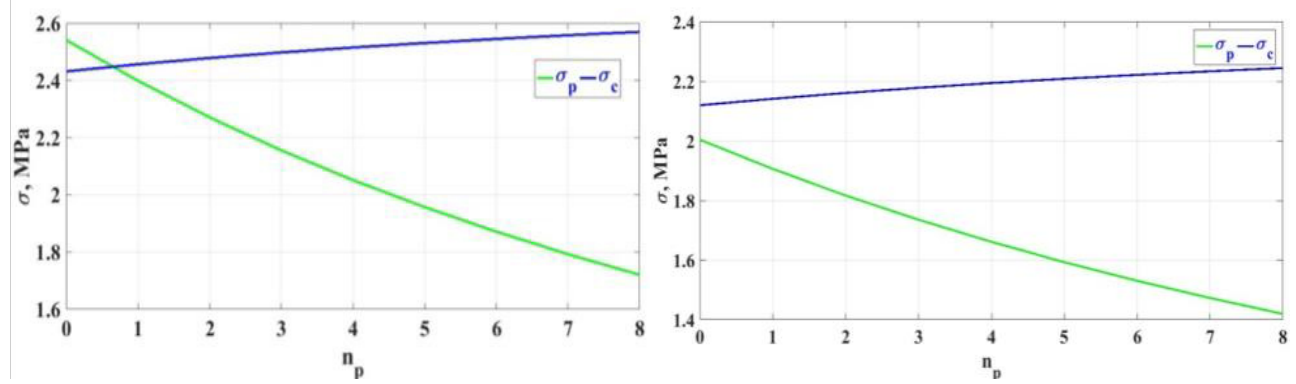

Fig. 6. Stresses of a bimodule t-beam on an elastic base depending on the width $b_{\mathrm{p}}$ of the bottom shelf $E_{\mathrm{p}}=5000 \mathrm{MPa}, E_{\mathrm{c}}=2250 \mathrm{MPa}: b_{\mathrm{p}}=28 \mathrm{~cm}$ (left), $b_{\mathrm{p}}=34 \mathrm{~cm}$ (right)

\section{Conclusions}

The proposed method makes it possible to analyze the influence of the location and number of reinforcement bars on the normal stresses arising in the cross-sections of the I-beam and the t-beam on the elastic base, taking into account and without taking into account the bimodularity of the material, as well as the dependence of the stress-strain state on the shape of the cross-section. 
The given numerical studies show a significant impact on the qualitative and quantitative picture of the stress-strain state reinforced beam on the elastic base when taking into account the heterogeneity of the material, which suggests the need to take into account this property of concrete in the calculations of the strength and stiffness of the elements of building structures.

\section{References}

1. A.N. Beskopylny, A.A. Veremeenko, B.M. Yazyev, MATEC Web of Conferences 106, 04004 (2017)

2. A.N. Beskopylny, I.G. Kadomtsev, N.I. Beskopylnaya, Theoretical foundations of civil engineering, 83-84 (2005)

3. A.N. Beskopylny, A.A. Veremeenko, E.E. Kadomtseva, N.I. Beskopylnaya MATEC Web of Conferences 129, 02046 (2017)

4. S.A. Ambartsumyan, Multi-Modulus theory of elasticity (Moscow, Science, 1982)

5. Z. Rigbi, ASME Journal of engineering materials and technology 102, 183-384 (1980)

6. A. N. Beskopylny, E.E. Kadomtseva, G.P. Strelnikov, Y.A. Berdnik, IOP Conf. Ser.: Earth Environ. Sci. 90, 012064 (2017)

7. A. N. Beskopylny, E.E. Kadomtseva, G.P. Strelnikov IOP Conf. Ser.: Earth Environ. Sci., 90, 012017 (2017)

8. A.N. Beskopylny, A.A. Lyapin, V.I. Andreev MATEC Web of Conferences 117, 00018 (2017)

9. A. Beskopylny, A. Lyapin, M. Kadomtsev, A. Veremeenko MATEC Web of Conferences 146, 02013 (2018)

10. A.S. Chepurnenko, V.I. Andreev, B.M. Yazyev, A.N. Beskopylny, MATEC Web of Conferences 67, 06059 (2016) 